\title{
Narrativa
}

\section{Emanuele BROCCIO, Tra amore e Resistenza. La drammaturgia di Beppe Fenoglio}

\author{
Elvira M. Ghirlanda
}

\section{OpenEdition}

\section{Journals}

Edizione digitale

URL: https://journals.openedition.org/narrativa/560

DOI: $10.4000 /$ narrativa. 560

ISSN: 2804-1224

\section{Editore}

Presses universitaires de Paris Nanterre

\section{Edizione cartacea}

Data di pubblicazione: 1 décembre 2018

Paginazione: $177-178$

ISBN: 978-2-84016-325-1

ISSN: $1166-3243$

\section{Notizia bibliografica digitale}

Elvira M. Ghirlanda, «Emanuele вRoccio, Tra amore e Resistenza. La drammaturgia di Beppe Fenoglio», Narrativa [Online], 40 | 2018, online dal 01 novembre 2021, consultato il 08 décembre 2021. URL: http://journals.openedition.org/narrativa/560 ; DOI: https://doi.org/10.4000/narrativa.560

Questo documento è stato generato automaticamente il 8 décembre 2021

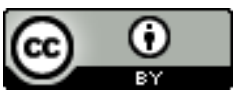

Narrativa est mise à disposition selon les termes de la Licence Creative Commons Attribution 4.0 International. 


\title{
Emanuele BROCCIO, Tra amore e Resistenza. La drammaturgia di Beppe Fenoglio
}

\author{
Elvira M. Ghirlanda
}

\section{NOTIZIA}

Emanuele BRoccio, Tra amore e Resistenza. La drammaturgia di Beppe Fenoglio, Bologna, Il Mulino, 2018, 126 p.

1 Il volume nasce dall'urgenza di trattare monograficamente l'intera produzione teatrale di Beppe Fenoglio e di sottrarla alla zona di marginalità in cui la critica aveva collocato tale scrittura come "esperimento".

2 Condotta nelle intimità dei testi, l'indagine si sviluppa cronologicamente e si suddivide in tre capitoli: il primo e il secondo sono dedicati rispettivamente alla Voce nella tempesta e a Serenate a Bretton Oaks (le cui stesure sono state terminate tra il 1947 e il 1948), il terzo accoglie il biennio 1960-1962, quindi Atto unico, Prologo [e], Lo sbandato.

Senza trascurare le problematiche filologiche dei testi e i puntuali riferimenti agli studi precedenti, l'analisi mira alla descrizione sia dei rapporti intessuti tra i testi dall'autore albese e la letteratura anglo-americana, sia delle relazioni tra le cinque pièces e il corpus della stessa prosa fenogliana. Nitidi si distinguono gli assi che legano La voce nella tempesta con Cime tempestose di Brontë (passando per la contaminazione del film di Wyler, omonimo al testo fenogliano e a questo di poco precedente), Serenate a Bretton Oaks con Piccola città di Wilder e con l'Antologia di Spoon River di Lee Masters, le tre pièces partigiane (Atto unico, Prologo [e], Lo sbandato) con i due romanzi della Resistenza Il partigiano Johnny e Una questione privata. Parallelamente la disamina converge nella messa a fuoco dello sviluppo di due temi dominanti, trasversali al macrotesto dell'autore, l'amore e la Resistenza. 
4 La restituzione è duplice. Innanzitutto, una precisa prospettiva comparatistica traccia il dialogismo aperto da Fenoglio e quel vitale movimento dialettico tra fedeltà e "fraintendimento" che diviene forza propulsiva di significanza - in termini riffaterriani - e che permette di scoprire l'autore come incantato e tormentato, "condannato a imparare i propri desideri più struggenti attraverso la consapevolezza dell'esserci di altri io" (cfr. Harold Bloom, L'angoscia dell'influenza. Una teoria della poesia).

5 Inoltre, Broccio compie in questo suo lavoro una salda integrazione tra scrittura drammaturgica e prosastica, mostrandone completo dominio e svelando come anzi “è nell'ipertesto teatrale che spesso l'ipotesto narrativo trova sviluppo o persino correzione" (p. 11). Una dinamica di cui lo studioso offre due interessanti emblemi: l'incapacità dei personaggi maschili di sottrarsi al triangolo amoroso e, quindi, di compiersi come soggetto; e il personaggio dai tratti pirandelliani del partigiano Califfo (Lo Sbandato) che lontano dalla dimensione eroica della Resistenza consegna la misura domestica di una crisi di identità storica ed esistenziale. 